\title{
Ultrafast Transient Absorption Spectrum of the Room Temperature Ionic Liquid 1-Hexyl-3-MethylImidazolium Bromide: Confounding Effects of Photo-degradation
} Raluca M. Musat, ${ }^{a,{ }^{*}}$ Robert A. Crowell, ${ }^{a}$ Dmitriy E. Polyanskiy, ${ }^{a}$ Marie F. Thomas, ${ }^{a, \dagger}$ James F. Wishart, ${ }^{a}$ Yosuke Katsumura, ${ }^{b}$ Kenji Takahashi, ${ }^{c, t}$

a Chemistry Department, Brookhaven National Laboratory, P.O. Box 5000 Upton, New York 11973, USA

b Department of Nuclear Engineering and Management, School of Engineering, The University of Tokyo, 7-3-1 Hongo, Bunkyo-ku, Tokyo 113-8656, Japan c Institute of Science and Engineering, Kanazawa University, Kakuma-machi, Kanazawa 920-1192, Japan

‡ Kenji Takahashi ktkenji@staff.kanazawa-u.ac.jp, Tel: +81(0)76-234-4828; Fax: $+81(0) 76-234-4829$

\begin{abstract}
The photochemistry of the charge transfer (CT) band of the room temperature ionic liquid (RTIL) 1-hexyl-3-methylimidazolium bromide $\left(\mathrm{HMIm}^{+} / \mathrm{Br}^{-}\right)$is investigated using near-IR to Vis ultrafast transient absorption (TA) and steady-state UV absorption spectroscopies. Continuous irradiation of the CT band at $266 \mathrm{~nm}$ results in the formation of photo-products that absorb strongly at $266 \mathrm{~nm}$. It is shown that these photo-products, which are apparently very stable, adversely affect ultrafast TA measurements. Elimination of these effects reveals at least two transient species that exist within the TA detection window of 100 fs to $3 \mathrm{~ns}$ and $500 \mathrm{~nm}$ to $1250 \mathrm{~nm}$. One of the components is a short-lived ( $<1 \mathrm{ps}$ ) species that absorbs at $1080 \mathrm{~nm}$. A second band exhibits a multicomponent spectrum that is very broad with an absorption maximum around $600 \mathrm{~nm}$ and a lifetime that is longer than the $3 \mathrm{~ns}$ window of our TA spectrometer. Within the signal to noise ratio of the TA spectrometer little to no solvated electron is generated by the CT mechanism.
\end{abstract}

\footnotetext{
* Present address: Institute of Science and Engineering, Kanazawa University, Kakuma-machi, Kanazawa 920-1192, Japan.

${ }^{\dagger}$ Present address: Department of Natural Science, Fordham University, New York, NY, 10023, USA.
} 
KEYWORDS: ionic liquid, charge transfer, excess electron, solvated electron, photo-degradation, ultrafast transient absorption spectroscopy. 


\section{INTRODUCTION ${ }^{\S}$}

Due to their unique characteristics: low vapor pressures, wide electrochemical windows and remarkable solvent properties, room temperature ionic liquids (RTILs) have been proposed for use in a wide variety of industrial applications.(Castner and Wishart 2010; Plechkova and Seddon 2008; Wishart 2009) Accordingly, RTILs have received considerable attention in both fundamental and applied research over the past decade. One class of these compounds, the imidazolium salts, has been shown to have numerous potential uses in many areas, especially in energy related technologies such as photoelectrochemical cells,(Grätzel 2001) nuclear fuel reprocessing,(Harmon, Smith, and Costa 2001) energy storage devices,(Simon and Gogotsi 2008) separation science(Luo et al. 2006; Pandey 2006) and carbon dioxide sequestration.(Gurkan et al. 2010; Jutz, Andanson, and Baiker 2011) Two issues that are common to almost all of the potential applications of imidazolium based ILs are the need to understand the fundamental aspects of the initial physico-chemical processes that involve charge transfer (CT) from the anion to cation and the solvation structure and dynamics of the anion (this is especially important for the use of ILs in separations and solar cell applications).(Dupont and Suarez 2006; Maroncelli 1993; Maroncelli et al. 2012)

Unfortunately, this class of ILs has been shown to be unstable under exposure to UV,(Brands, Chandrasekhar, and Unterreiner 2007; Katoh and Takahashi 2009) ionizing radiation(Berthon et al. 2006; Le Rouzo et al. 2009) and elevated

\footnotetext{
§ ABBREVIATIONS

RTILs, Room Temperature Ionic Liquids

CT, charge transfer

CTTS, charge transfer to solvent

TA, transient absorption

DFT, density functional theory

$\mathrm{HMIm}^{+} / \mathrm{Br}^{-}$, 1-hexyl-3-methyl imidazolium bromide

$\mathrm{HMIm}^{+} / \mathrm{NTf}_{2}^{-}$, 1-hexyl-3-methyl imidazolium bis(trifluoromethanesulfonyl)imide

$\mathrm{BMIM}^{+} / \mathrm{Cl}^{-}$, 1-butyl-3-methylimidazolium chloride

$\mathrm{BMIm}^{+} / \mathrm{PF}_{6}^{-}$, 1-butyl-3-methylimidazolium hexa-fluoro-phosphate

$\mathrm{BMIm}^{+} / \mathrm{BF}_{4}^{-}$, 1-butyl-3-methylimidazolium tetra-fluoro-borate

$\mathrm{TMPA}^{+} / \mathrm{NTf}_{2}^{-}, N, N, N$-trimethyl-n-propylammonium bis(trifluoromethanesulfonyl)imide

[X: $\left.\mathrm{e}^{-}\right]$, halogen:electron pair
} 
temperatures.(Awad et al. 2004; Del Sesto et al. 2009) In order to design robust ILs that can fulfill their potential applications it is necessary to study the fundamental processes that lead to their degradation which begin with the dynamics of the initial CT process.

As a result there have been numerous ultrafast studies of halide-based imidazolium ionic liquids (for a recent review see reference (Nese Chandrasekhar and Unterreiner 2010) and references therein).(Nese Chandrasekhar and Unterreiner 2010) In particular is the pioneering work of the Unterreiner group using femtosecond transient absorption (TA) spectroscopy to study the ultrafast dynamics following excitation of the CT band of a series of chloride- and iodide-based imidazolium ILs.(Brands, Chandrasekhar, and Unterreiner 2007; N Chandrasekhar, Endres, and Unterreiner 2006; N. Chandrasekhar, Schalk, and Unterreiner 2008; Nese Chandrasekhar and Unterreiner 2010) The CT band was excited at $266 \mathrm{~nm}$ and probed over the region of 555 -1556 nm. For the halide-based imidazolium salts they observed an instrumentresponse-limited rise followed by a bi-exponential decay with time constants of about 2 ps and several hundred picoseconds. The spectra taken at 400 fs (iodide) and 10 ps (chloride) after excitation exhibit a very broad $(>1 \mathrm{eV})$ absorption peak that is centered near $1500 \mathrm{~nm}$. They attributed the fast component to a caged halogen:electron pair ([X:e-]) and the longer lived specie to a solvated electron. They suggested that one possibility to analyze the results was in terms of the Staib and Borgis(Staib and Borgis 1996) model in which the initial excitation immediately generates the metastable $\left[\mathrm{X}: \mathrm{e}^{-}\right]$pair. The electron either escapes to become solvated or recombines with the halogen atom or a neighboring imidazolium ring. As an alternative explanation they offered the possibility that the excess electrons are generated via ionization of the imidazolium to form a dication. However, as they 
point out it has been shown that their excitation wavelength of $257 \mathrm{~nm}(4.8 \mathrm{eV})$ is not energetic enough to ionize the imidazolium cation. During the course of their measurements they observed that both the iodide- and chloride-based imidazolium salts “turn yellow upon continuous irradiation”. Based upon steady-state UV absorption measurements they attributed the yellow color to the formation of di- and tri-halide species. Furthermore, they go on to show that the TA kinetics observed at a single wavelength strongly depend upon the scan rate, suggesting possible contributions from stable photo-products.(Brands, Chandrasekhar, and Unterreiner 2007) In earlier work(Shkrob et al. 2013) part of our team observed that in dilute solutions of $\mathrm{C}_{n} \mathrm{MIm}^{+} / \mathrm{Br}^{-}$in $\mathrm{C}_{n} \mathrm{MIm}^{+} / \mathrm{NTf}_{2}{ }^{-}$, the released halogen atom very rapidly abstracts $\mathrm{H}$ from the aliphatic arms of the IL cations, forming $\mathrm{HBr}$, the dimer radical, $\mathrm{C}_{2}{ }^{\cdot+}$, and the $\mathrm{H}$-loss radical, $\mathrm{C}(-\mathrm{H})^{+\bullet}$. Consequently, the dihalide or trihalide radicals are not produced under these conditions.

In this letter we focus on the photochemistry of the anion-to-cation CT process in the RTIL 1-hexyl-3-methyl imidazolium bromide $\left(\mathrm{HMIm}^{+} / \mathrm{Br}^{-}\right)$. We show that the interpretations of previous ultrafast studies(Brands, Chandrasekhar, and Unterreiner 2007; N Chandrasekhar, Endres, and Unterreiner 2006; N. Chandrasekhar, Schalk, and Unterreiner 2008; Nese Chandrasekhar and Unterreiner 2010) are questionable due to the rapid buildup of stable photo-products that possess a strong absorption cross section at the pump wavelength $(266 \mathrm{~nm})$ and also exhibit emission in the visible region (the emission goes away when the sample is refreshed). By eliminating the effects of photo-degradation, new absorption features connected to the initial photochemical events in imidazolium based RTILs are revealed and it now becomes possible to begin to unravel these complex processes. The new results indicate that the initial CT events may include contributions from excited states, caged 
halogen:electron pairs, and radical cation dimers. The experimental details can be found in the supplemental information section.

\section{MATERIALS AND METHODS}

\subsection{Sample preparation}

All chemicals used were of commercial grade and used without purification: 1Methylimidazole and 1-bromohexane were purchased from Sigma Aldrich; lithium bis(trifluoromethylsulfonyl)imide was obtained from IoLiTech; acetonitrile and ethyl acetate were purchased from Mallinckrodt Chemicals. Ultrapure Millipore Milli-Q water with a resistivity of $18.2 \mathrm{M} \Omega$ and less than $10 \mathrm{ppb}$ organic carbon was used. $\mathrm{HMIm}^{+} / \mathrm{Br}^{-}$and $\mathrm{HMIm}^{+} / \mathrm{NTf}_{2}{ }^{-}$, with the structures presented in Figure 1, were synthesized according to the method presented in the Supplementary Information.

The sample cell used for the steady state measurements is a $12 \mu \mathrm{m}$ pathlength commercial (Harrick) IR cell using $2 \mathrm{~mm}$ thick $\mathrm{CaF}_{2}$ windows. The transient absorption measurements use the same cell with a $12 \mu \mathrm{m}$ pathlength and $56 \mu \mathrm{m}$ pathlength when flowing the sample. The samples were prepared under controlled Ar atmosphere in order to avoid contamination or hydration. For the transient absorption measurement, a dual pump syringe (New Era Pump Systems Inc.) system is used to refresh the sample, which allows continuous flow of the sample at a $2 \mathrm{ml} / \mathrm{min}$ rate. During the steady state and transient absorption measurements, without sample flow, a fast emission buildup is observed, which increases with the exposure time; this emission disappears when flowing the samples.

Details about the steady state and transient absorption measurements are presented in the Supplementary Information. 

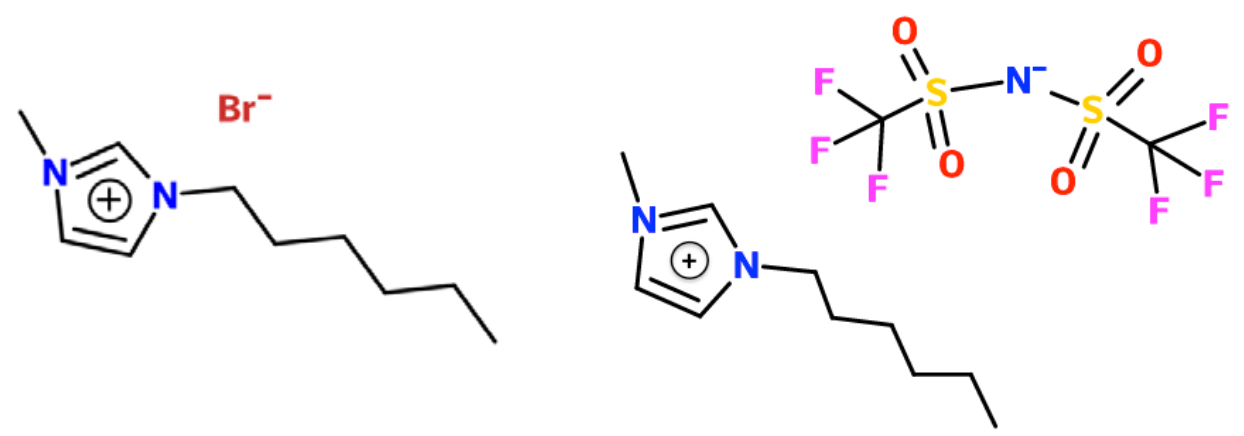

Figure 1. Molecular structure of the $\mathrm{HMIm}^{+} / \mathrm{Br}^{-}$(left image) and $\mathrm{HMIM}^{+} / \mathrm{NTf}_{2}^{-}$(right image).

\section{RESULTS AND DISCUSSION}

Static spectra under different 266 nm irradiation exposures are shown in Figure 2. For comparison, the dashed line is the spectrum of the bromide-free RTIL 1-hexyl-3methyl imidazolium bis(trifluoromethanesulfonyl)imide $\left(\mathrm{HMIm}^{+} / \mathrm{NTf}_{2}^{-}\right)$without UV photolysis. Based on the similarity to imidazole, the absorption below $230 \mathrm{~nm}$ (peak at $210 \mathrm{~nm}$ ) is assigned to the $\pi-\pi^{*}$ transition originating from the $\mathrm{C}=\mathrm{C}$ bond of the imidazolium cation(Katoh 2007) (note: $\left[\mathrm{NTf}_{2}{ }^{-}\right]$absorbs at $\lambda<210 \mathrm{~nm}$ ). Substitution of $\left[\mathrm{NTf}_{2}^{-}\right]$with bromide produces an additional absorption feature that has been shown to be a CT band.(Katoh, Hara, and Tsuzuki 2008) Figure 2 shows the buildup of the absorption in the UV as a function of exposure to $266 \mathrm{~nm}, 60$ ps pulses from the fourth harmonic of a $10 \mathrm{~Hz} \mathrm{Nd:YAG} \mathrm{laser}\left(90 \mathrm{~mJ} / \mathrm{cm}^{2}\right)$. In order to evaluate the effect of the UV irradiation on the sample, the UV-Vis spectrum was collected after every 10 laser shots at a dose of $90 \mathrm{~mJ} / \mathrm{cm}^{2}$ per pulse, up to a total dose of $486 \mathrm{~J} / \mathrm{cm}^{2}$. The spectra presented in Figure 2 were collected before and after 200, 1800, 4500, 4800 and 5400 laser shots. Upon UV exposure there is a rapid buildup of absorption at $266 \mathrm{~nm}$, similar to what has been previously observed.(Brands, Chandrasekhar, and Unterreiner 2007; Katoh and Takahashi 2009) The inset of Figure 2 shows that the 
buildup of this absorption at $266 \mathrm{~nm}$ is linear with respect to the exposure. Additionally there is the appearance of a new broad absorption band that extends to $\lambda$ $>400 \mathrm{~nm}$. Simultaneous to the growth of this band a decrease in the CT band absorbance occurs. After a few seconds of exposure the color of the sample begins to change. The $\mathrm{HMIm}^{+} / \mathrm{Br}^{-}$takes on a yellow color which becomes more pronounced as the exposure time is increased, similar to what has been previously reported for both photolysis(Brands, Chandrasekhar, and Unterreiner 2007) and gamma radiolysis(Qi et al. 2007; Yuan et al. 2009) of imidazolium based ILs. Concurrent to this, the samples begin to exhibit a visible emission. This emission has been previously observed upon UV irradiation(Katoh and Takahashi 2009) and its properties are not characterized in this study. The emission does not appear when the sample is continuously refreshed during irradiation. Eventually the formation of bubbles is observed and the samples turn black. The bubbles have been shown to be mainly hydrogen and hydrocarbons while the 'black crude' that eventually forms is a complex mix of polymers.(Le Rouzo et al. 2009)

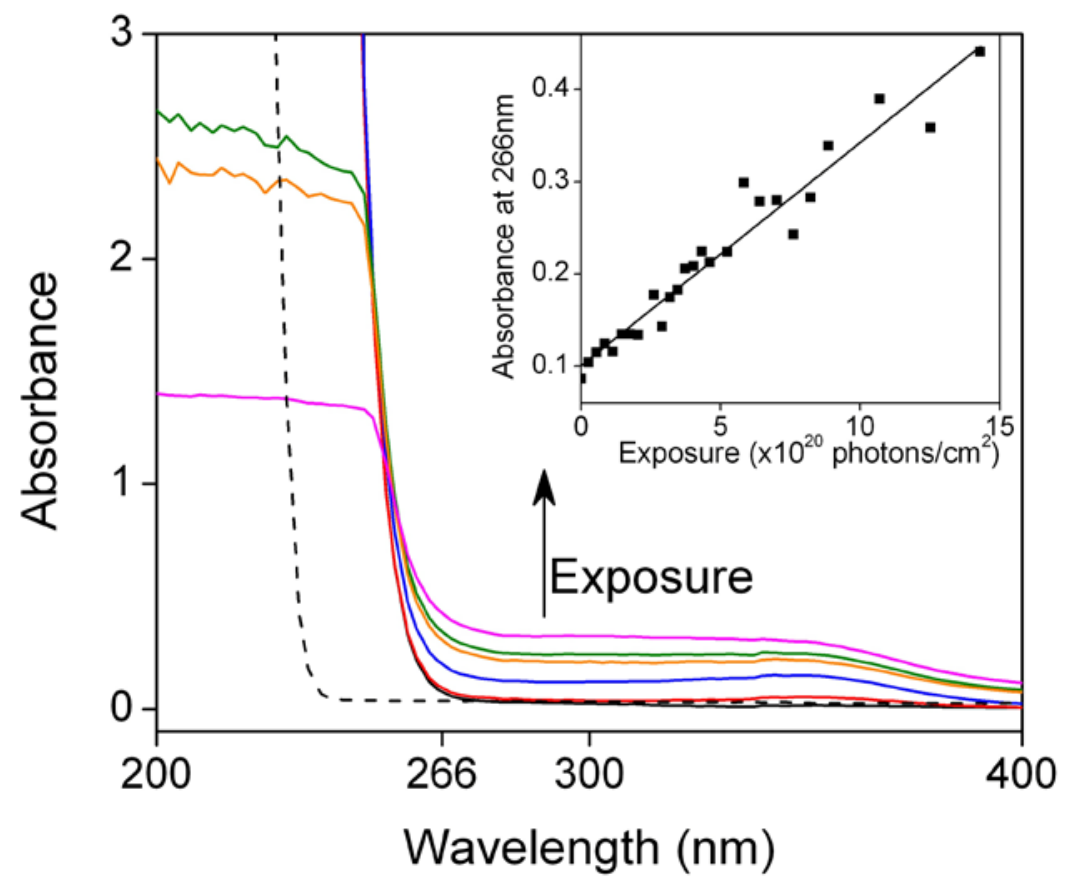


Figure 2. Steady state absorption spectrum of $\mathrm{HMIm}^{+} / \mathrm{Br}^{-}$as a function of laser irradiation at $266 \mathrm{~nm}$. The dashed line is the spectrum of HMIm ${ }^{+} / \mathrm{NTf}_{2}^{-}$(no bromide, no UVirradiation), which shows that the laser is being absorbed by the CT band. The black spectrum corresponds to no UV irradiation while each additional spectrum corresponds to 200, 1800, 4500, 4800 and 5400 laser shots at $10 \mathrm{~Hz}\left(90 \mathrm{~mJ} / \mathrm{cm}^{2}\right.$ pulse). The inset shows the buildup of absorption at $266 \mathrm{~nm}$ as a function of exposure is linear.

Figure 3 shows the effect of the degradation on the TA spectrum. Figure $3 a$ is the TA spectrum for a static sample and Figure 3b shows the TA spectrum for a sample that is continuously refreshed via raster scanning and flowing (note the difference in the absorbance scales). It is estimated that the cumulative photon flux is about 100150 times smaller for the sample that is refreshed (see the Supporting Information for details). There are striking differences between the two TA spectra. Both show a broad band in the near IR and a peak near $600 \mathrm{~nm}$; however, the $600 \mathrm{~nm}$ peak in the visible clearly shows different spectral and temporal characteristics. Additionally, the relative absorption strengths for the visible and near IR bands are strikingly different between the two TA spectra. 

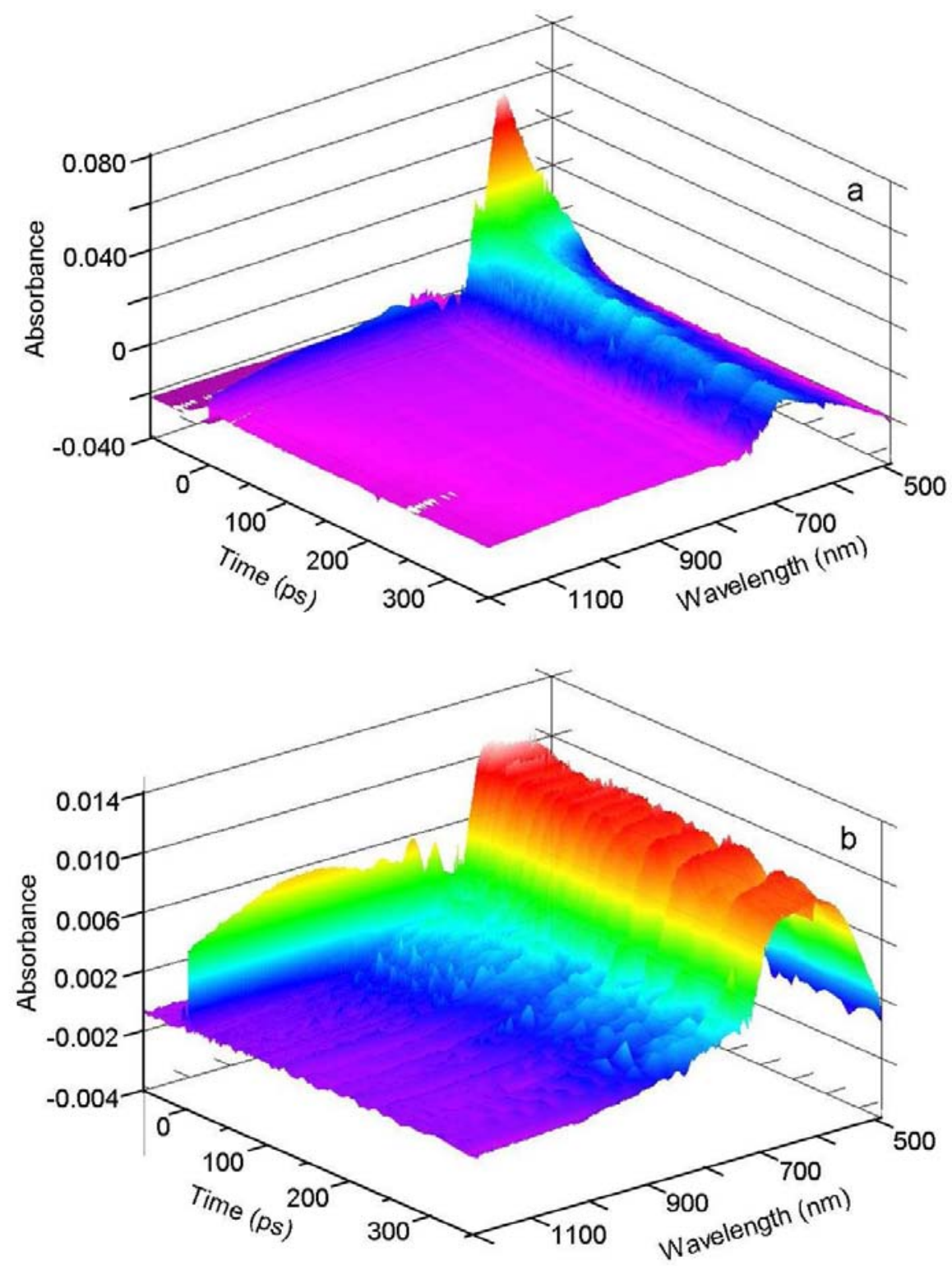

Figure 3. a. The TA spectrum for a static sample of $\mathrm{HMIm}^{+} / \mathrm{Br}^{-}$. b. The TA spectrum of $\mathrm{HMIm}^{+} / \mathrm{Br}^{-}$in the sample that was refreshed. The cumulative photon flux is about 100-150 times less for the refreshed sample.

Figure 4 shows an expanded view of the near-IR peaks under static (4a) and refreshed conditions (4b), and dramatic differences are observed in this region as well. Both static and refreshed samples show a broad band that has a rapid decay. Results of a bi-exponential fit to both data sets are shown in Table 1. However, as 
shown in Figure 5, the time-resolved absorbance decay at $1250 \mathrm{~nm}$ for the refreshed sample goes almost completely to zero (to within the signal-to-noise ratio of our TA apparatus), whereas there is a significant long-lived component present in the static sample. Also for the refreshed sample, qualitatively, the spectrum has a well-defined shape that peaks at $1080 \mathrm{~nm}$. The noise at longer times that is observed in Figure 3b and Figure $4 \mathrm{~b}$ is due to mechanical noise from the raster scanning and the peaks at time zero around $800 \mathrm{~nm}$ are due to interference from the Ti:Sapphire laser fundamental.

$$
\Delta \mathrm{OD}(\text { Normalized })=\mathrm{A}_{0}+\mathrm{A}_{1} \exp \left[-\mathrm{t} / \tau_{1}\right]+\mathrm{A}_{2} \exp \left[-\mathrm{t} / \tau_{2}\right]
$$



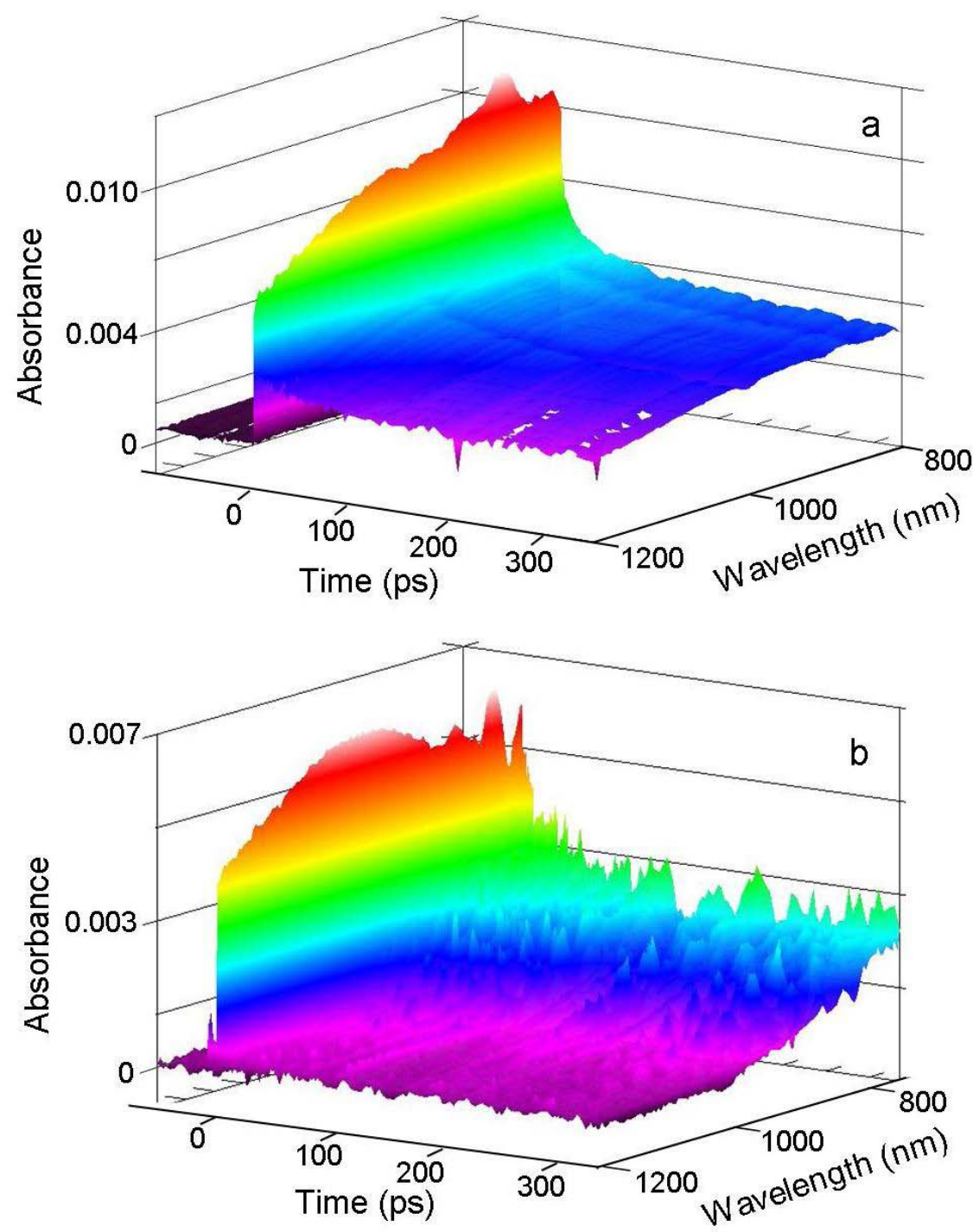

Figure 4. The same as Figure 3 with a wavelength range of $800 \mathrm{~nm}$ to $1250 \mathrm{~nm}$ to highlight the features in the near-IR. The peaks at time zero around $800 \mathrm{~nm}$ are due to interference from the laser fundamental. 


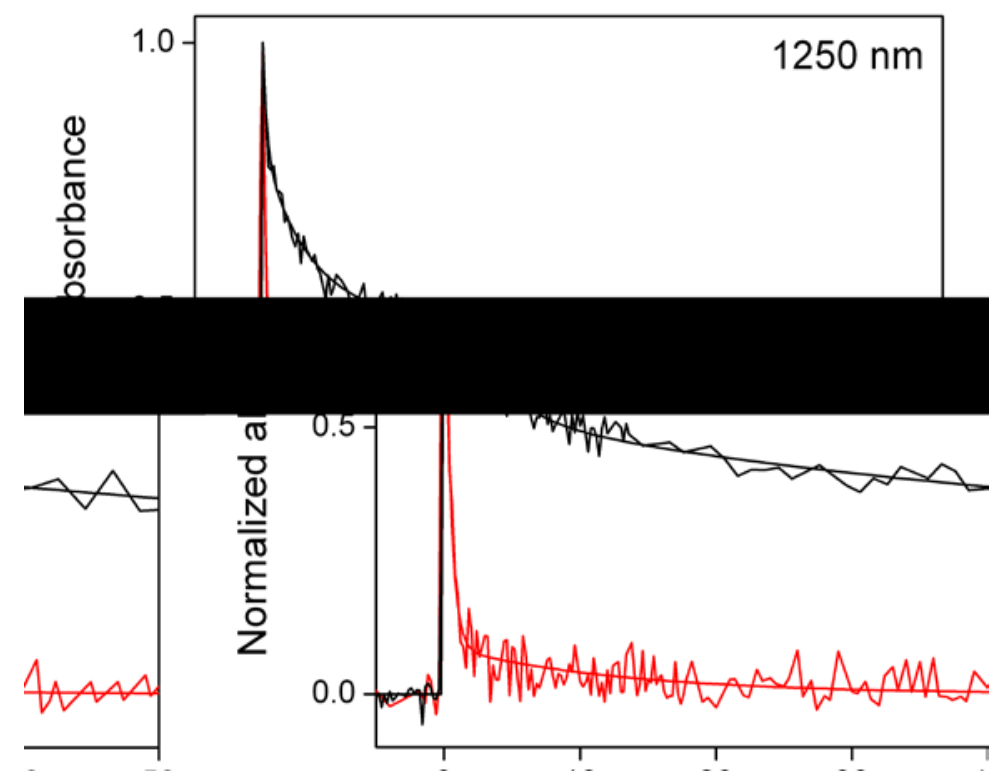

Figure 5. Normalized 2-D absorbance-time traces results at $1250 \mathrm{~nm}$ along with biexponential fits for static (black) and continuously refreshed (red) samples. The results of the biexponential fits are shown in Table 1.

Table 1. Biexponential fits for absorbance decays at 1250nm in $\mathrm{HMIm}^{+} / \mathrm{Br}^{-}$:

\begin{tabular}{|l|c|c|c|c|}
\hline Sample & $\mathrm{A}_{1}$ & $\tau_{1}(\mathrm{ps})$ & $\mathrm{A}_{2}$ & $\tau_{2}(\mathrm{ps})$ \\
\hline Static & $0.19 \pm 0.01$ & $0.30 \pm 0.04$ & $0.26 \pm 0.03$ & $>3 \times 10^{3}$ \\
\hline Refreshed & $0.90 \pm 0.03$ & $0.43 \pm 0.03$ & $0.09 \pm 0.02$ & $12 \pm 3$ \\
\hline
\end{tabular}


Insight to the identification of the photo-products of Figure 2 can be gained by looking at previous pulse radiolysis studies. Grodkowski and Neta(Grodkowski and Neta 2002) used pulse radiolysis to study the reactions of the $\mathrm{Br}_{2}{ }^{-}$radical anion in ILs. They identified a spectral feature at $360 \mathrm{~nm}$ that they assigned to the $\mathrm{Br}_{2}{ }^{-}$radical anion. In water the extinction coefficient of $\mathrm{Br}_{2}^{-}$is around $10 \times 10^{3} \mathrm{M}^{-1} \mathrm{~cm}^{-1}$. Using the pulse-accelerated-flow technique, Wang et al(T. X. Wang et al. 1994) reported that the extinction coefficient for aqueous $\mathrm{Br}_{3}{ }^{-}$to be in the range of $25 \times 10^{3} \mathrm{M}^{-1} \mathrm{~cm}^{-1}$ at 266 $\mathrm{nm}$ to $39 \times 10^{3} \mathrm{M}^{-1} \mathrm{~cm}^{-1}$ at $270 \mathrm{~nm}$. It is expected that the extinction coefficients should not be much different in $\mathrm{HMIm}^{+} / \mathrm{Br}^{-}$. The formation of $\mathrm{Br}_{2}^{-}$and $\mathrm{Br}_{3}{ }^{-}$is expected in $\mathrm{HMIm}^{+} / \mathrm{Br}^{-}$, where the concentration of $\mathrm{Br}^{-}$is around $5 \mathrm{M}$. Additionally, due to the high viscosity of $\mathrm{HMIm}^{+} / \mathrm{Br}^{-}$(ca. 10,000 cP) these species should be very stable. Pulse radiolysis studies by Behar et al(Behar, Gonzalez, and Neta 2001) on a series of 1-butyl-3-methylimidazolium $\left(\mathrm{BMIm}^{+}\right)$salt solutions (1-butyl-3-methylimidazolium chloride, $\quad \mathrm{BMIm}^{+} / \mathrm{Cl}^{-}$, 1-butyl-3-methylimidazolium hexafluorophosphate, $\mathrm{BMIm}^{+} / \mathrm{PF}_{6}{ }^{-}$, and 1-butyl-3-methylimidazolium tetrafluoroborate, $\mathrm{BMIm}^{+} / \mathrm{BF}_{4}^{-}$, show a strong absorption band at $323 \mathrm{~nm}$ with an extinction coefficient of $5.9 \times 10^{3} \mathrm{M}^{-1} \mathrm{~cm}^{-1}$. They show that $\left(\mathrm{BMIm}^{+}\right)$is rapidly reduced by the solvated electron to produce BMIm (rate constant $1.9 \times 10^{10} \mathrm{M}^{-1} \mathrm{~cm}^{-1}$ ) and assign the band at $323 \mathrm{~nm}$ to the BMIm radical.

In a recent study, Shkrob and Wishart (Shkrob et al. 2013; Wishart and Shkrob 2009) used a combination of computational chemistry methods, low-temperature electron spin resonance and TA spectroscopy to study electron localization in imidazolium-based ILs. They propose that the electron localization on the imidazolium cation produces a gauche dimer radical cation $\left(\mathrm{C}_{2}^{+\bullet}\right)$ via reaction of the $\mathrm{BMIm}^{\bullet}$ radical with a BMIm${ }^{+}$cation, and that these species should absorb in the near- 
IR and the visible to near-UV regions. They suggest that one and two photon excitation of bromide anions in IL lead to formation of bromine atoms that quickly abstracts $\mathrm{H}$ from the alkyl chains of the IL cation; in two photon excitation, the 2imidazolyl radical generated via the charge transfer promptly eliminates the alkyl arm. The implications of these results are very interesting. They suggest that in imidazolium ILs the result of electron localization could be the formation of radical ions rather than neutral radicals, and the implications here are that the initial CT chemistry could be dominated by radical ion reactions.

Therefore, some of the possible photo-products that would interfere with the TA measurements are $\mathrm{Br}_{2}{ }^{-}, \mathrm{Br}_{3}{ }^{-}, \mathrm{C}_{2}{ }^{+\bullet}$, imidazolium radical and various polymers formed by the $\mathrm{C}-\mathrm{H}$ bond fissure. Because we do not know the relative yields and extinction coefficients of the possible photo-products (at $266 \mathrm{~nm}$ ) we are unable to estimate their concentration, but clearly, for the degraded samples, they dominate the absorption at $266 \mathrm{~nm}$. We conclude that previous ultrafast TA experiments on halide-based imidazolium RTILs were primarily exciting the photo-products rather than inducing CT in ILs.

\section{CONCLUSIONS}

In the present work we do not find strong evidence for the formation by CT of a solvated electron having broad absorption in the near-IR ( 1500 nm) as observed by the Unterreiner group.(Brands, Chandrasekhar, and Unterreiner 2007) In the photolysis work of Katoh et al(Katoh et al. 2007) on the charge transfer to solvent (CTTS) excitation of excess iodide in the RTIL $N, N, N$-trimethyl- $n-$ propylammonium[NTf $\left.{ }_{2}^{-}\right]\left(\mathrm{TMPA}^{+} / \mathrm{NTf}_{2}^{-}\right)$, a broad absorbance band centered at about $1250 \mathrm{~nm}$ was assigned to the solvated electron. It is important to note that in these experiments they are not investigating the CT process but the CTTS process from 
excess iodide to the IL. Similarly, it may be possible that the broad band observed on the picosecond time scale by the Unterreiner group is indeed a solvated electron. However the solvated electron they observe generated by CTTS of the photo-products rather than by the CT process.

Based on previous femto and picosecond photolytic studies on CTTS in aqueous halides(Lian et al. 2006) and picosecond radiolytic investigations in concentrated acid solutions(Ma et al. 2014), the most probable assignment of the band at $1080 \mathrm{~nm}$ is to the formation of a caged halogen:electron pair. The exact fate of this caged halogen:electron pair is the topic of current work. Because this band is so short-lived it is most likely not $\mathrm{C}_{2}^{+\bullet}$. As far as the multicomponent band in the visible region, this could be due to the rapid formation of $\mathrm{C}_{2}^{+\bullet}$. It has even been suggested that the visible band could arise from triplets of the imidazolium core that are excited from excess energy of the CT.(Shkrob n.d.) In addition, ab initio molecular dynamic simulations and DFT calculations(Z. Wang et al. 2009) suggest that on picosecond time scales, excess electrons in imidazolium halide ILs (chloride, specifically) perturb the electronic structure of neighboring imidazolium cations, possibly giving rise to the observed electronic transitions.

Future work using a variety of techniques will be undertaken to understand the origin of this band. We conclude that the interpretation of previous studies of CT in ILs is questionable due to the rapid formation of stable photo-products that have very strong absorption at the pump wavelengths used. Elimination of the photo-products reveals new bands in the near-IR and visible regions. Future work is focused on making assignments to these bands so that we can better understand the photochemistry associated with CT in RTILs. 


\section{AKNOWLEDGEMENTS}

The authors thank Dr. I. A. Shkrob for insightful comments. This work was supported by the U.S. Department of Energy, Office of Basic Energy Sciences, Division of Chemical Sciences, Geosciences and Biosciences under contracts \# DEAC02-98CH10886 and DE-SC0012704.

\section{References}

Awad, Walid H., Jeffrey W. Gilman, Marc Nyden, Richard H. Harris, Thomas E. Sutto, John Callahan, Paul C. Trulove, Hugh C. DeLong, and Douglas M. Fox. 2004. "Thermal Degradation Studies of Alkyl-Imidazolium Salts and Their Application in Nanocomposites.” Thermochimica Acta 409: 3-11.

Behar, David, Carlos Gonzalez, and Pedatsur Neta. 2001. "Reaction Kinetics in Ionic Liquids: Pulse Radiolysis Studies of 1-Butyl-3-Methylimidazolium Salts.” The Journal of Physical Chemistry A 105(32): 7607-14. http://dx.doi.org/10.1021/jp011405o.

Berthon, L, S I Nikitenko, I Bisel, C Berthon, M Faucon, B Saucerotte, N Zorz, and $\mathrm{Ph}$ Moisy. 2006. "Influence of Gamma Irradiation on Hydrophobic RoomTemperature Ionic Liquids [BuMeIm]PF6 and [BuMeIm](CF3SO2)2N.” Dalton Transactions: 2526-34.

Brands, H, N Chandrasekhar, and A-N Unterreiner. 2007. "Ultrafast Dynamics of Room Temperature Ionic Liquids after Ultraviolet Femtosecond Excitation.” Journal of Physical Chemistry B 111(18): 4830-36.

Castner, Edward W., and James F. Wishart. 2010. "Spotlight on Ionic Liquids.” Journal of Chemical Physics 132: 120901.

Chandrasekhar, N, F Endres, and A-N Unterreiner. 2006. "Evidence for LaserInduced Formation of Solvated Electrons in Room Temperature Ionic Liquids.” Physical Chemistry Chemical Physics 8: 3192-96.

Chandrasekhar, N., O. Schalk, and A.-N. Unterreiner. 2008. "Femtosecond UV Excitation in Imidazolium-Based Ionic Liquids." Journal of Physical Chemistry B 112(49): 15718-24. 
Chandrasekhar, Nese, and Andreas-Neil Unterreiner. 2010. "Photochemical Processes in Ionic Liquids on Ultrafast Timescales.” Physical Chemistry Chemical Physics 12: $1698-1708$.

Dupont, Jairton, and Paulo A Z Suarez. 2006. "Physico-Chemical Processes in Imidazolium Ionic Liquids.” Physical Chemistry Chemical Physics 8: 2441-52.

Grätzel, M. 2001. "Photoelectrochemical Cells.” Nature 414: 338-44.

Grodkowski, J, and P Neta. 2002. "Reaction Kinetics in the Ionic Liquid Methyltributylammonium Bis(Trifluoromethylsulfonyl)imide . Pulse Radiolysis Study of $\cdot \mathrm{CF}_{3}$ Radical Reactions.” The Journal of Physical Chemistry A 106: 5468-73.

Gurkan, B., B. F. Goodrich, E. M. Mindrup, L. E. Ficke, M. Massel, S. Seo, T. P. Senftle, H. Wu, M. F. Glaser, J. K. Shah, E. J. Maginn, J. F. Brennecke, and W. F. Schneider. 2010. "Molecular Design of High Capacity, Low Viscosity, Chemically Tunable Ionic Liquids for $\mathrm{CO}_{2}$ Capture.” The Journal of Physical Chemistry Letters 1: 3494-99. http://dx.doi.org/10.1021/jz101533k.

Harmon, Chuck D., Wayne H. Smith, and David A. Costa. 2001. "Criticality Calculations for Plutonium Metal at Room Temperature in Ionic Liquid Solutions.” Radiation Physics and Chemistry 60: 157-59.

Jutz, Fabian, Jean Michel Andanson, and Alfons Baiker. 2011. "Ionic Liquids and Dense Carbon Dioxide: A Beneficial Biphasic System for Catalysis.” Chemical Reviews 111: 322-53.

Katoh, Ryuzi. 2007. “Absorption Spectra of Imidazolium Ionic Liquids.” Chemistry Letters 36(10): 1256-57.

Katoh, Ryuzi, Mikiya Hara, and Seiji Tsuzuki. 2008. "Ion Pair Formation in [bmim]I Ionic Liquids.” The Journal of Physical Chemistry B 112(48): 15426-30.

Katoh, Ryuzi, and Kenji Takahashi. 2009. "Photo-Degradation of Imidazolium Ionic Liquids.” Radiation Physics and Chemistry 78(12): 1126-28. http://dx.doi.org/10.1016/j.radphyschem.2009.07.002.

Katoh, Ryuzi, Yoichi Yoshida, Yosuke Katsumura, and Kenji Takahashi. 2007. "Electron Photodetachment from Iodide in Ionic Liquids through ChargeTransfer-to-Solvent Band Excitation.” The Journal of Physical Chemistry. B 111(18): 4770-74. http://dx.doi.org/10.1021/jp067107e.

Lian, Rui, Dmitri A. Oulianov, Robert A. Crowell, Ilya A. Shkrob, Xiyi Chen, and Stephen E. Bradforth. 2006. "Electron Photodetachment from Aqueous Anions. 3. Dynamics of Geminate Pairs Derived from Photoexcitation of Mono- Vs Polyatomic Anions.” The Journal of Physical Chemistry A 110(29): 9071-78.

Luo, H, S Dai, P V Bonnesen, T J Haverlock, B A Moyer, and A C III Buchanan. 2006. "A Striking Effect of Ionic- Liquid Anions in the Extraction of Sr2+ and 
Cs+ by Dicyclohexano- 18- Crown- 6." Solvent Extraction and Ion Exchange 24(1).

Ma, Jun, Schmidhammer, Uli, Mostafavi, Mehran. 2014. "Direct Evidence for Transient Pair Formation between a Solvated Electron and $\mathrm{H}_{3} \mathrm{O}^{+}$Observed by Picosecond Pulse Radiolysis." The Journal of Physical Chemistry Letters 5: 2219-2223.

Maroncelli, Mark. 1993. "The Dynamics of Solvation in Polar Liquids.” Journal of Molecular Liquids 57: 1-37.

Maroncelli, Mark, Xin-Xing Zhang, Min Liang, Durba Roy, and Nikolaus P Ernsting. 2012. "Measurements of the Complete Solvation Response of Coumarin 153 in Ionic Liquids and the Accuracy of Simple Dielectric Continuum Predictions." Faraday Discussions 154: 409-24.

Pandey, Siddharth. 2006. "Analytical Applications of Room-Temperature Ionic Liquids: A Review of Recent Efforts.” Analytica Chimica Acta 556: 38-45.

Plechkova, Natalia V, and Kenneth R Seddon. 2008. "Applications of Ionic Liquids in the Chemical Industry.” Chemical Society Reviews 37: 123-50.

Qi, Mingying, Guozhong Wu, Shimou Chen, and Yaodong Liu. 2007. "Gamma Radiolysis of Ionic Liquid 1-Butyl-3-Methylimidazolium Hexafluorophosphate.” Radiation Research 167(5): 508-14.

Le Rouzo, G, C Lamouroux, V Dauvois, A Dannoux, S Legand, D Durand, P Moisy, and G Moutiers. 2009. "Anion Effect on Radiochemical Stability of RoomTemperature Ionic Liquids under Gamma Irradiation.” Dalton Transactions (31): 6175-84.

Del Sesto, Rico E., Mark T. McCleskey, Clay Macomber, Kevin C. Ott, Andrew T. Koppisch, Gary A. Baker, and Anthony K. Burrell. 2009. "Limited Thermal Stability of Imidazolium and Pyrrolidinium Ionic Liquids.” Thermochimica Acta 491: 118-20.

Shkrob, Ilya A. "Private Communication.”

Shkrob, Ilya A., Timothy W. Marin, Robert A. Crowell, and James F. Wishart. 2013. "Photo- and Radiation-Chemistry of Halide Anions in Ionic Liquids." Journal of Physical Chemistry A 117: 5742-56.

Simon, Patrice, and Yury Gogotsi. 2008. "Materials for Electrochemical Capacitors.” Nature Materials 7: 845-54.

Staib, Arnulf, and Daniel Borgis. 1996. "Reaction Pathways in the Photodetachment of an Electron from Aqueous Chloride: A Quantum Molecular Dynamics Study.” The Journal of Chemical Physics 104(22): 9027-39. 
Wang, Tian Xiang, Mark D Kelley, John N Cooper, Richard C Beckwith, and Dale W Margerum. 1994. "Equilibrium Kinetic and Uv Spectral Charac of Aque Bromine Chloride-Bromine-and Chlorine Species.” Inorganic Chemistry 33(25): 5872-78.

Wang, Zhiping, Liang Zhang, Xiaohua Chen, Robert I Cukier, and Yuxiang Bu. 2009. "Excess Electron Solvation in an Imidazolium-Based Room-Temperature Ionic Liquid Revealed by Ab Initio Molecular Dynamics Simulations.” The Journal of Physical Chemistry. B 113(24): 8222-26. http://dx.doi.org/10.1021/jp902575s.

Wishart, James F, and Ilya A Shkrob. 2009. "The Radiation Chemistry of Ionic Liquids and Its Implications for Their Use in Nuclear Fuel Processing.” In Ionic Liquids: From Knowledge to Application, eds. R. D. Rogers, N. V. Plechkova, and K. R. Seddon. Washington D.C.: American Chemical Society, 119-34.

Wishart, James F. 2009. "Energy Applications of Ionic Liquids." Energy \& Environmental Science 2(9): 956. http://dx.doi.org/10.1039/b906273d.

Yuan, Liyong, Jing Peng, Ling $\mathrm{Xu}$, Maolin Zhai, Jiuqiang Li, and Genshuan Wei. 2009. "Radiation-Induced Darkening of Ionic Liquid [C4mim][NTf2] and Its Decoloration.” Radiation Physics and Chemistry 78: 1133-36. 